\title{
A hidden classical symmetry of QCD
}

\author{
L. Ya. Glozman ${ }^{1, \star}$
}

${ }^{1}$ Institute of Physics, University of Graz, A-8010 Graz, Austria

\begin{abstract}
The classical part of the QCD partition function (the integrand) has, ignoring irrelevant exact zero modes of the Dirac operator, a local $S U\left(2 N_{F}\right) \supset S U\left(N_{F}\right)_{L} \times$ $S U\left(N_{F}\right)_{R} \times U(1)_{A}$ symmetry which is absent at the Lagrangian level. This symmetry is broken anomalously and spontaneously. Effects of spontaneous breaking of chiral symmetry are contained in the near-zero modes of the Dirac operator. If physics of anomaly is also encoded in the same near-zero modes, then their truncation on the lattice should recover a hidden classical $S U\left(2 N_{F}\right)$ symmetry in correlators and spectra. This naturally explains observation on the lattice of a large degeneracy of hadrons, that is higher than the $S U\left(N_{F}\right)_{L} \times S U\left(N_{F}\right)_{R} \times U(1)_{A}$ chiral symmetry, upon elimination by hands of the lowestlying modes of the Dirac operator. We also discuss an implication of this symmetry for the high temperature QCD.
\end{abstract}

\section{Introduction}

The QCD Lagrangian in Minkowski space-time has in the chiral limit the chiral symmetry:

$$
U\left(N_{F}\right)_{L} \times U\left(N_{F}\right)_{R}=S U\left(N_{F}\right)_{L} \times S U\left(N_{F}\right)_{R} \times U(1)_{A} \times U(1)_{V} .
$$

In the following we will always drop the $U(1)_{V}$ symmetry that is irrelevant to our subject. The $U(1)_{A}$ symmetry is invariance of the Lagrangian upon the axial flavor-neutral transformation

$$
\Psi(x) \rightarrow e^{i \alpha \gamma_{5}} \Psi(x) ; \quad \bar{\Psi}(x) \rightarrow \bar{\Psi}(x) e^{i \alpha \gamma_{5}} .
$$

The $S U\left(N_{F}\right)_{L} \times S U\left(N_{F}\right)_{R}$ symmetry implies invariance under a pure flavor rotation $S U\left(N_{F}\right)$ ( for $N_{F}=2$ it is the isospin symmetry) as well as invariance under the axial flavor transformation

$$
\Psi(x) \rightarrow e^{i \gamma_{5} \frac{\vec{\lambda} \cdot \vec{x}}{2}} \Psi(x) ; \quad \bar{\Psi}(x) \rightarrow \bar{\Psi}(x) e^{i \gamma_{5} \frac{\vec{k} \cdot \vec{\alpha}}{2}}
$$

where $\vec{\lambda}$ are $S U\left(N_{F}\right)$ generators.

The axial $U(1)_{A}$ symmetry is broken anomalously, which is due to a noninvariance of the integration measure in the functional integral under a local $U(1)_{A}$ transformation [1]. The $S U\left(N_{F}\right)_{A}$ "symmetry"1 (3) is broken spontaneously, because the ground state of the theory, the vacuum, is not invariant under the transformation (3). The latter noninvariance is encoded in the nonzero quark condensate of the vacuum, $<0|\bar{\Psi}(x) \Psi(x)| 0>\neq 0$. The quark condensate of the vacuum can be expressed

\footnotetext{
^e-mail: leonid.glozman@uni-graz.at

${ }^{1}$ These transformations do not form a closed subgroup of the chiral group.
} 
through a density of the near-zero modes $\lambda \rightarrow 0$ of the Euclidean Dirac operator (the Banks-Casher relation [2])

$$
\lim _{m \rightarrow 0}<0|\bar{\Psi}(x) \Psi(x)| 0>=-\pi \rho(0) .
$$

The idea of the $N_{F}=2$ lattice studies [3,4] was to understand what would happen with $J=1,0$ mesons upon truncation of the near-zero modes of the Dirac operator. One expects that after truncation correlators of operators that are connected with each other through the $S U(2)_{A}$ transformation would become identical. If hadrons survive this truncation, then masses of chiral partners should be equal. It has turned out that a very clean exponential decay of correlators was detected in all $J=1$ mesons. This implies that confined bound states survive the truncation. In the $J=0$ mesons, while all $J=0$ correlators become identical, the ground states disappear, because there is no exponential decay of the corresponding correlators: The near-zero modes are crucially important for existence of the (pseudo) Goldstone bosons, which is not surprising.

It has also turned out that the truncation restores in hadrons not only the $S U(2)_{L} \times S U(2)_{R}$ symmetry but also the $U(1)_{A}$ symmetry. One then concludes that the same lowest-lying modes are responsible for both $S U(2)_{L} \times S U(2)_{R}$ and $U(1)_{A}$ breakings which is consistent with the instanton-induced mechanism of both breakings [7-10].

However, a larger degeneracy that includes all possible chiral multiplets of the $J=1$ mesons was detected, which was completely unexpected. This surprising degeneracy implies a symmetry that is higher than the $S U(2)_{L} \times S U(2)_{R} \times U(1)_{A}$. This not yet known symmetry has been reconstructed in refs. $[11,12]$ and turned out to be

$$
S U\left(2 N_{F}\right) \supset S U\left(N_{F}\right)_{L} \times S U\left(N_{F}\right)_{R} \times U(1)_{A} .
$$

Transformations of this group include both the flavor rotations of the left- and right-handed quarks as well as transformations that mix the left- and right-handed components. This symmetry has been confirmed in lattice simulations with the $J=2$ mesons [5] and in baryons [6].

While such an enlarged symmetry, that is larger than the symmetry of the QCD Lagrangian, was observed in the lattice experiment, its origin was mysterious. The light on this issue has been shed in ref. [13]. It has been shown that the classical part of the QCD partition function has, excluding irrelevant exact zero modes, a $S U\left(2 N_{F}\right)$ local symmetry. This symmetry is not a symmetry of the Euclidean QCD Lagrangian because the irrelevant exact zero modes of the Euclidean Dirac operator break it. Consequently, we refer it as a hidden classical symmetry. This hidden classical symmetry is broken by the anomaly to $S U\left(N_{F}\right)_{L} \times S U\left(N_{F}\right)_{R}$. Spontaneous breaking of chiral symmetry reduces it to $S U\left(N_{F}\right)_{V}$. Effects of spontaneous and anomalous breakings are encoded in the near-zero modes of the Dirac operator. Consequently, truncation on the lattice of the lowest-lying modes restores the hidden $S U\left(2 N_{F}\right)$ symmetry, which naturally explains degeneracies observed in lattice experiment.

Now we will present some details.

\section{What can be apriori expected from the low-mode truncation?}

A truncation of the low-lying modes of the Dirac operator means the following. The Euclidean Lagrangian with $N_{F}$ degenerate quarks in a given gauge background is:

$$
\mathcal{L}=\Psi^{\dagger}(x)\left(\gamma_{\mu} D_{\mu}+m\right) \Psi(x)
$$

where 


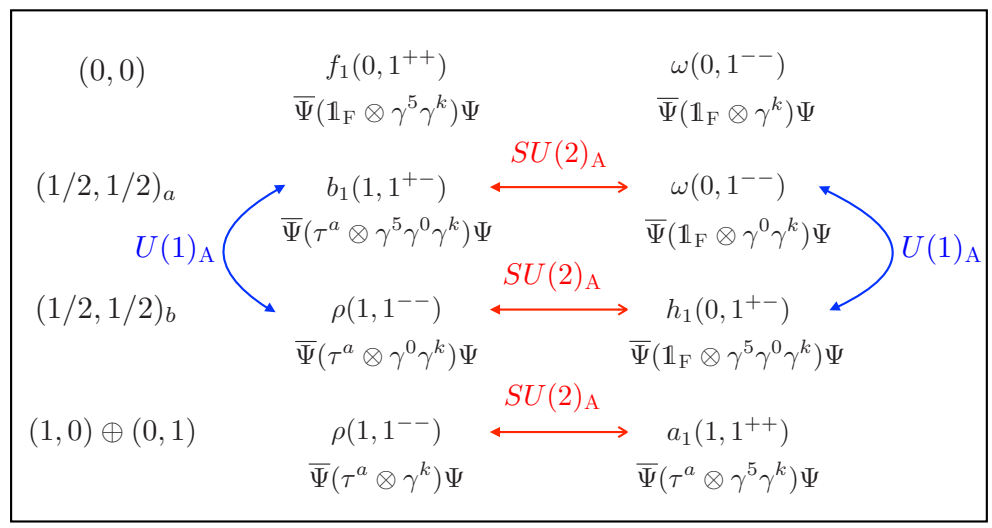

Figure 1. $S U(2)_{L} \times S U(2)_{R}$ and $U(1)_{A}$ classification of the $J=1$ meson operators.

$$
D_{\mu}=\partial_{\mu}+i g \frac{t^{a}}{2} A_{\mu}^{a}
$$

The hermitian Dirac operator, $i \gamma_{\mu} D_{\mu}$, has in a volume $V$ a discrete spectrum with real eigenvalues $\lambda_{n}$ :

$$
i \gamma_{\mu} D_{\mu} \Psi_{n}(x)=\lambda_{n} \Psi_{n}(x) \text {. }
$$

We subtract from the full quark propagator $S_{F u l l}$ the lowest $k$ eigenmodes of the Dirac operator

$$
S(x, y)=S_{\text {Full }}(x, y)-\sum_{n=1}^{k} \frac{1}{\lambda_{n}+i m} \Psi_{n}(x) \Psi_{n}^{\dagger}(y) .
$$

At the same time we keep the gauge configurations intact. Given these truncated quark propagators we apply standard procedures to extract hadron spectra using the variational approach. Then we study dependence of hadron masses on the truncation number $k$. We perform $N_{F}=2$ dynamical lattice calculations with the overlap Dirac operator with the gauge configurations generated by the JLQCD collaboration, for details see refs. [3-6].

When the near-zero modes, that are responsible for spontaneous chiral symmetry breaking are subtracted we can expect the $S U(2)_{L} \times S U(2)_{R}$ symmetry in correlators. If in addition the hadron states survive this surgery, then we can expect a mass degeneracy of chiral partners. For the $J=1$ mesons the chiral partners are linked by the red arrows on Fig. 1.

The $U(1)_{A}$ transformation connects operators that are linked by the blue arrows on Fig. 1. Consequently, if the whole chiral symmetry of QCD $S U(2)_{L} \times S U(2)_{R} \times U(1)_{A}$ is restored, then we can expect a degeneracy of four mesons from the $(1 / 2,1 / 2)_{a}$ and $(1 / 2,1 / 2)_{b}$ representations on the one hand, and on the other hand a degeneracy of the $\rho$ and $a_{1}$ mesons from the $(1,0)+(0,1)$ chiral representation. Note that in the chirally symmetric world there two independent orthogonal $\rho$-mesons that belong to two different chiral representations. In the real world with chiral symmetry breaking these two chiral representations are mixed in the meson wave function and two different $\rho$ operators couple to one and the same $\rho$-meson.

The $S U(2)_{L} \times S U(2)_{R} \times U(1)_{A}$ symmetry does not connect, however, the four mesons from the $(1 / 2,1 / 2)_{a}$ and $(1 / 2,1 / 2)_{b}$ representations with the other mesons in Fig. 1. Consequently, given only 

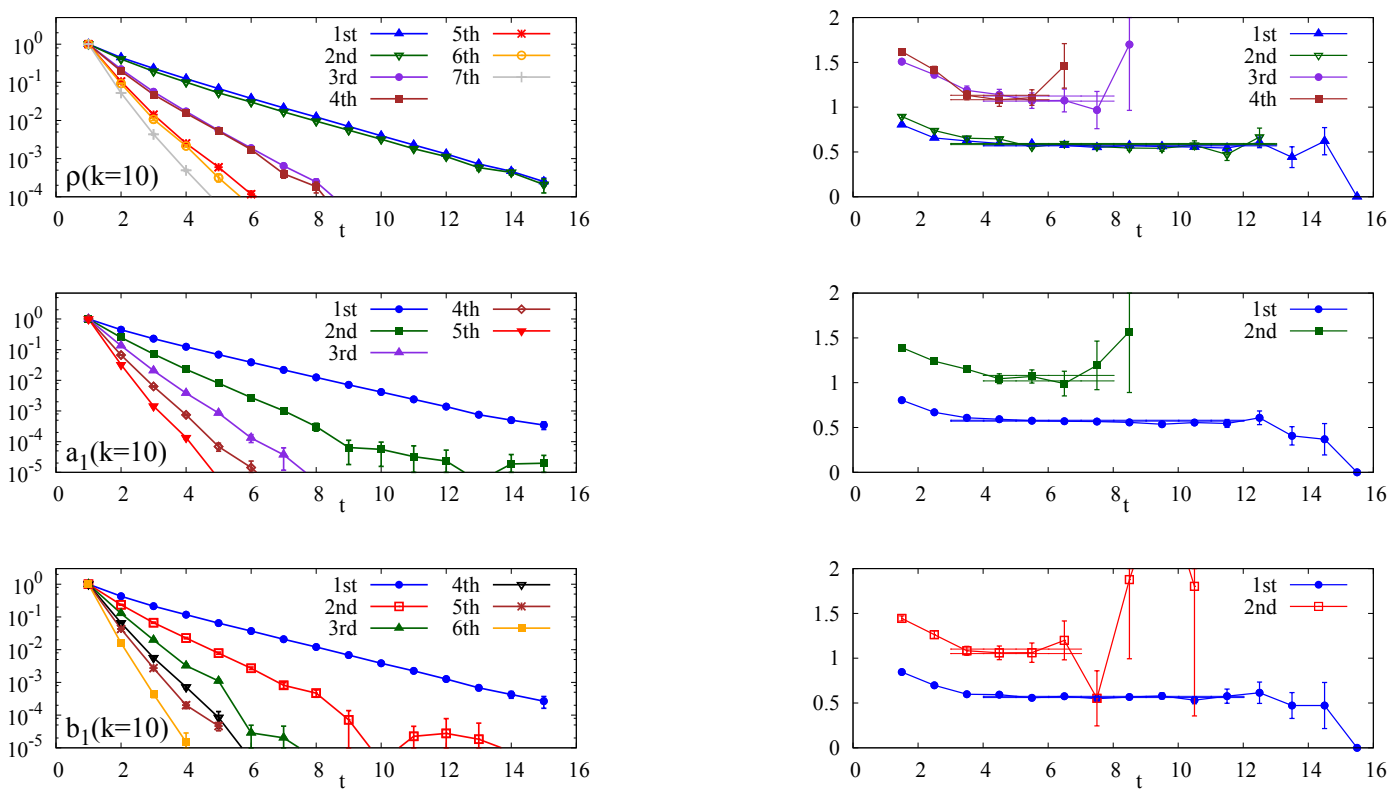

Figure 2. The eigenvalues of the cross-correlation matrix and effective mass plateaus for the isovector $J=1$ mesons with $k=10$

the $S U(2)_{L} \times S U(2)_{R} \times U(1)_{A}$ chiral symmetry we cannot expect a degeneracy larger than it is shown by arrows on Fig. 1 .

\section{Results}

We do not show here our results for the real world $(k=0)$, since they are typical for all lattice studies and the experimental meson spectra are reasonably reproduced. The results for the eigenvalues of the cross-correlation matrix and effective mass plateau at $k=10$ (10 lowest Dirac eigenmodes have been removed) for the isovector mesons are shown in Fig. 2.

A very clean exponential decay of the correlators is obvious, which means that there are physical states. It is much cleaner than in the untruncated (real) world. The reason for this is intuitively clear: After truncation there are no pion fluctuations in the system. We can conclude that mesons (which are bound states now) survive the truncation.

Note a double degeneracy of the rho-meson eigenvalues, which is absent in the untruncated world. This double degeneracy can be obtained only if both rho-operators from Fig. 1 are used in the crosscorrelation matrix. This double degeneracy tells that there are two independent orthogonal degenerate $\rho$-mesons. If we put two lowest rho-eigenvalues, the lowest $a_{1}$ and the lowest $b_{1}$ eigenvalues on the same plot, then we will see that they all are identical (the same is true with the higher eigenvalues, but the result is less precise). This means that there is a symmetry in the system that is higher than $S U(2)_{L} \times S U(2)_{R} \times U(1)_{A}$.

Evolution of meson masses is shown in Fig. 3. We clearly see a larger degeneracy than the chiral $S U(2)_{L} \times S U(2)_{R} \times U(1)_{A}$ symmetry of the QCD Lagrangian. What does it mean?! The same results persist for the $J=2$ mesons and baryons. 


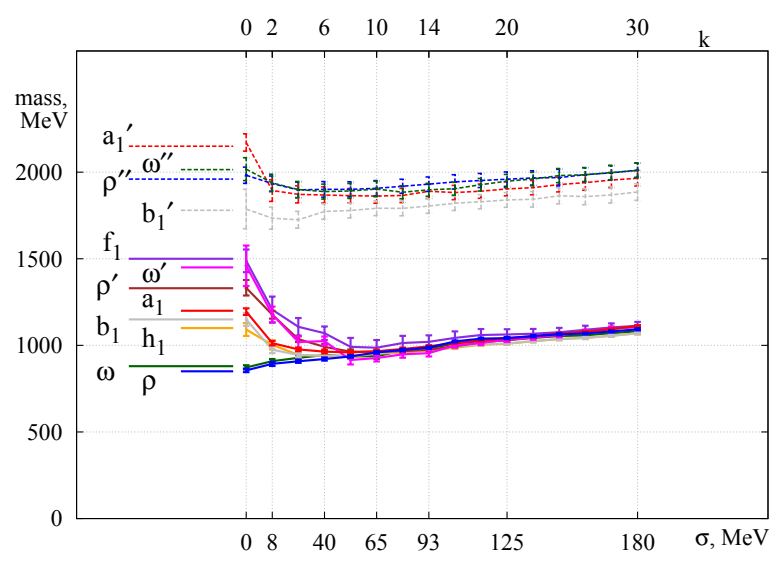

Figure 3. $J=1$ meson mass evolution as a function of the truncation number $k$. $\sigma$ shows energy gap in the Dirac spectrum.

\section{$4 S U(4)$ symmetry of the meson spectra}

Given this degeneracy first we need to understand what symmetry group does it correspond. This unexpected new symmetry has been reconstructed in ref. [11].

Given the standard spin, parity, etc. quantum numbers we can construct explicitly basis vectors for all irreducible representations of the chiral group shown in Fig. 1.

(i) $(\mathbf{0 , 0})$ :

$$
|(0,0) ; \pm ; J\rangle=\frac{1}{\sqrt{2}}|\bar{R} R \pm \bar{L} L\rangle_{J}
$$

(ii) $(1 / 2,1 / 2)_{a}$ and $(1 / 2,1 / 2)_{b}$ :

$$
\begin{gathered}
\left|(1 / 2,1 / 2)_{a} ;+; I=0 ; J\right\rangle=\frac{1}{\sqrt{2}}|\bar{R} L+\bar{L} R\rangle_{J}, \\
\left|(1 / 2,1 / 2)_{a} ;-; I=1 ; J\right\rangle=\frac{1}{\sqrt{2}}|\bar{R} \vec{\tau} L-\bar{L} \vec{\tau} R\rangle_{J}, \\
\left|(1 / 2,1 / 2)_{b} ;-; I=0 ; J\right\rangle=\frac{1}{\sqrt{2}}|\bar{R} L-\bar{L} R\rangle_{J}, \\
\left|(1 / 2,1 / 2)_{b} ;+; I=1 ; J\right\rangle=\frac{1}{\sqrt{2}}|\bar{R} \vec{\tau} L+\bar{L} \vec{\tau} R\rangle_{J}
\end{gathered}
$$

(iii) $(\mathbf{0 , 1}) \oplus(1,0)$ :

$$
|(0,1)+(1,0) ; \pm ; J\rangle=\frac{1}{\sqrt{2}}|\bar{R} \vec{\tau} R \pm \bar{L} \vec{\tau} L\rangle_{J},
$$

Now we need to to find a minimal group that contains $S U(2)_{L} \times S U(2)_{R} \times U(1)_{A}$ as a subgroup and that combines all these vectors into one irreducible representation. These new symmetry transformations must connect all these basis vectors. The latter requirement can be achieved if these new symmetry transformations mix the leftand right-handed quarks. Consequently, the required symmetry group must contain as a subgroup the $S U(2)_{C S}$ chiralspin rotations that act on the following doublets: 


$$
\mathrm{U}=\left(\begin{array}{l}
u_{L} \\
u_{R}
\end{array}\right), \quad \mathrm{D}=\left(\begin{array}{l}
d_{L} \\
d_{R}
\end{array}\right) .
$$

A three-dimensional imaginary space where these rotations are performed is called the chiralspin space. The chiralspin rotations mix the right- and left-handed components of the fermion fields. It is similar to the well familiar isospin space: Rotations in the isospin space mix particles with different electric charges.

If we combine the $S U(2)_{C S}$ and the isospin $S U(2)$ group into one larger group one arrives at the $S U(4)$ group with the fundamental vector

$$
\Psi=\left(\begin{array}{l}
u_{\mathrm{L}} \\
u_{\mathrm{R}} \\
d_{\mathrm{L}} \\
d_{\mathrm{R}}
\end{array}\right) .
$$

The dim $=15$ irreducible representation of this group connects all $(0,0),(1 / 2,1 / 2)_{a},(1 / 2,1 / 2)_{b},(1,0)+(0,1)$ vectors. One of the $(0,0)$ basis vectors, namely $|(0,0) ;-; J=1\rangle>$ is a singlet of $S U(4)$.

We can construct an explicit realization of the $S U(2)_{C S}$ and $S U(4)$ algebra that acts on Dirac spinors [12]. Then the $S U(2)_{C S}$ chiralspin rotations are generated through

$$
\boldsymbol{\Sigma}=\left\{\gamma^{0}, i \gamma^{5} \gamma^{0},-\gamma^{5}\right\}, \quad\left[\Sigma^{i}, \Sigma^{j}\right]=2 i \epsilon^{i j k} \Sigma^{k} .
$$

The Dirac spinor transforms under a global or local $S U(2)_{C S}$ transformation as

$$
\Psi \rightarrow \Psi^{\prime}=e^{i \varepsilon \cdot \Sigma / 2} \Psi .
$$

The $S U(4)$ group contains at the same time $S U(2)_{L} \times S U(2)_{R}$ and $S U(2)_{C S} \supset U(1)_{A}$ and has the following set of generators:

$$
\left\{\left(\tau^{a} \otimes 1_{D}\right),\left(1_{F} \otimes \Sigma^{i}\right),\left(\tau^{a} \otimes \Sigma^{i}\right)\right\} .
$$

The global and local $S U(4)$ transformations of the Dirac spinor are defined through

$$
\Psi \rightarrow \Psi^{\prime}=e^{i \epsilon \cdot \mathbf{T} / 2} \Psi .
$$

The $S U(2)_{C S}$ and $S U(4)$ transformations of all operators from Fig. 1 are shown in Fig. 4.

\section{Zero modes and hidden classical symmetries of Euclidean QCD}

The $S U(4)$ symmetry is obtained in lattice simulations upon subtraction of the near-zero modes of the Dirac operator. It implies that this symmetry should be encoded in the Euclidean QCD. Obviously the Lagrangian (6) does not have this symmetry. This is because the Dirac operator does not commute with the $S U(2)_{C S}$ transformations. ${ }^{2}$ Then we should recover at which level this symmetry is hidden in Euclidean QCD. More explicitly, we have to find a part of the Euclidean QCD formalism that breaks this symmetry.

Consider the zero modes of the Dirac equation,

$$
\gamma_{\mu} D_{\mu} \Psi_{0}(x)=0 .
$$

Given standard antiperiodic boundary conditions for the quark field along the time direction, the zero modes are solutions of the Dirac equation with the gauge configurations of a nonzero global topological charge. The difference of numbers of the left-handed and right-handed zero modes is according to the Atiyah-Singer theorem fixed by the global topological charge $Q$ of the gauge configuration:

$$
n_{L}-n_{R}=Q .
$$

Some $S U(2)_{C S}$ transformations rotate the right-handed spinor into the left-handed one and vice versa. Consequently, the zero modes explicitly violate the $S U(2)_{C S}$ and $S U\left(2 N_{F}\right)$ symmetries: The zero modes introduce an

\footnotetext{
${ }^{2}$ In Euclidean space we have to substitute the $\gamma_{0}$ matrix through the $\gamma_{4}$ matrix.
} 


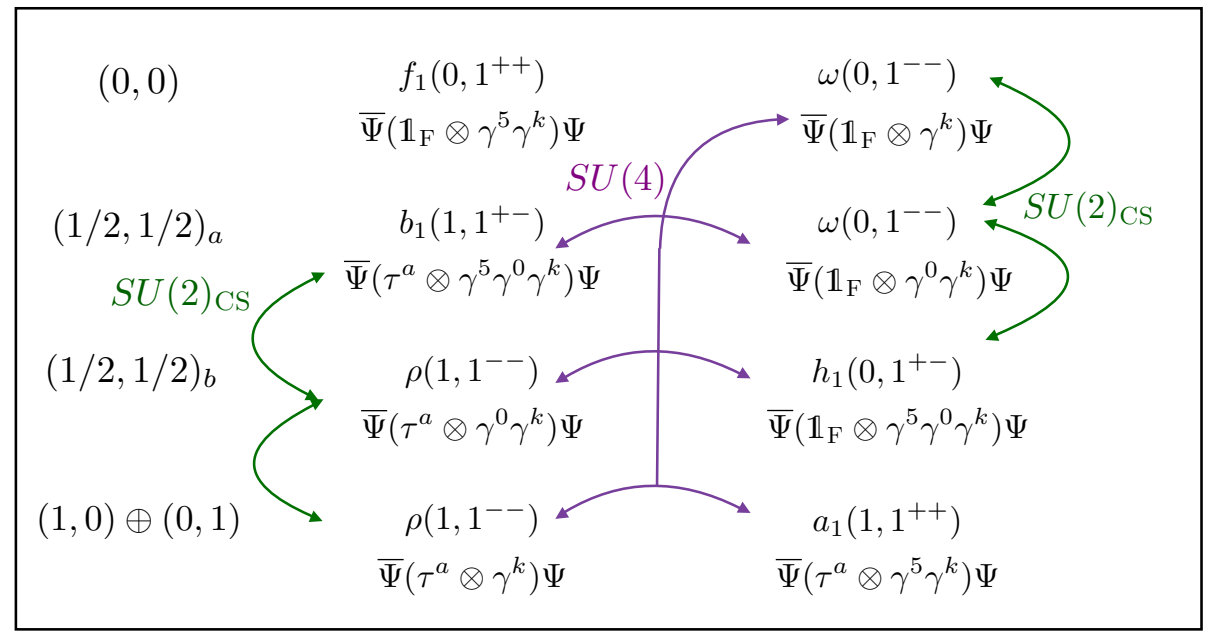

Figure 4. The green arrows connect operators that belong to the $S U(2)_{C S}$ triplets. The $f_{1}$ and $a_{1}$ operators are the $S U(2)_{C S}$ singlets. The purple arrows show the $S U(4) 15$-plet. The $f_{1}$ operator is a singlet of $S U(4)$.

asymmetry between the left- and right-handed degrees of freedom and break the $S U(2)_{C S}$ invariance. The latter is possible only if there is no asymmetry between the left and the right.

It is well understood, however, that the exact zero modes are completely irrelevant since their contributions to the Green functions and observables vanish in the thermodynamical limit $V \rightarrow \infty$ [14-16]. Consequently, in the finite volume calculations we can subtract the irrelevant exact zero modes.

We can expand fields $\Psi(x)$ and $\Psi^{\dagger}(x)$ in the Lagrangian over a complete and orthonormal set $\Psi_{n}(x)$ of the eigenvalue problem (8):

$$
\Psi(x)=\sum_{n} c_{n} \Psi_{n}(x), \quad \Psi^{\dagger}(x)=\sum_{k} \bar{c}_{k} \Psi_{k}^{\dagger}(x),
$$

where $\bar{c}_{k}, c_{n}$ are Grassmannian numbers. Then the fermionic part of the QCD partition function takes the following form

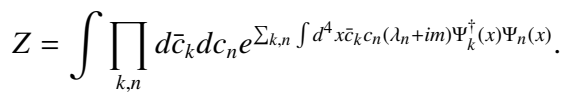

Now we can directly read-off symmetry properties of the classical part of the partition function, i.e. of the integrand. This functional contains only a superposition of terms $\Psi_{k}^{\dagger}(x) \Psi_{n}(x)$. It is precisely $S U(2)_{C S}$ and $S U\left(2 N_{F}\right)$ symmetric, because

$$
\left(U \Psi_{k}(x)\right)^{\dagger} U \Psi_{n}(x)=\Psi_{k}^{\dagger}(x) \Psi_{n}(x),
$$

where $U$ is any local or global transformation from the groups $S U(2)_{C S}$ and $S U\left(2 N_{F}\right), U^{\dagger}=U^{-1}$. The exact zero modes, for which the equation (24) does not hold, have been subtracted from the partition function. We conclude that classically the Euclidean QCD without the irrelevant exact zero mode contributions is invariant with respect to both global and local $S U(2)_{C S}$ and $S U\left(2 N_{F}\right)$ transformations.

The term "hidden classical $S U\left(2 N_{F}\right)$ symmetry" should be correctly understood. It is not a global symmetry of the Lagrangian and consequently there are no respective conserved Noether currents. However, it is a real local symmetry of the classical part of the QCD partition function ignoring irrelevant exact zero modes.

How is this hidden classical symmetry broken? The integration measure in (23) is not invariant under a local $U(1)_{A}$ transformation [1]. Consequently, the $U(1)_{A}$ anomaly breaks the classical $U(1)_{A}$ symmetry. Since 
the $U(1)_{A}$ is a subgroup of $S U(2)_{C S}$, the anomaly breaks either the $S U(2)_{C S}$ symmetry. Hence the classical $S U\left(2 N_{F}\right) \supset S U\left(N_{F}\right)_{L} \times S U\left(N_{F}\right)_{R} \times U(1)_{A}$ symmetry is broken by anomaly to $S U\left(N_{F}\right)_{L} \times S U\left(N_{F}\right)_{R}$.

The quark condensate in Minkowski space breaks all $U(1)_{A}, S U(2)_{C S}, S U\left(N_{F}\right)_{L} \times S U\left(N_{F}\right)_{R}$ and $S U\left(2 N_{F}\right)$ symmetries to the vector flavor symmetry $S U\left(N_{F}\right)_{V}$. Hence, the new hidden classical $S U(2)_{C S}$ and $S U\left(2 N_{F}\right)$ symmetries are broken both by the condensate and anomalously.

Spontaneous chiral symmetry breaking is encoded in the near-zero modes of the Dirac operator, as it follows from the Banks-Casher relation. If anomaly is also encoded in the near-zero modes, as suggested e.g. by the instanton mechanism of both breakings, then removal on lattice of the near-zero modes should restore not only chiral $S U\left(N_{F}\right)_{L} \times S U\left(N_{F}\right)_{R}$ and $U(1)_{A}$ symmetries, but also a larger $S U\left(2 N_{F}\right)$ symmetry, which naturally explains lattice observations reviewed in previous sections.

\section{What symmetries should one expect in mesons and baryons upon truncation of the near-zero modes?}

From the results presented in Fig. 3 it is clearly seen that the degeneracy pattern is larger than $S U(4)$, because the $S U(4)$ singlet $\left(f_{1}\right)$ and the $S U(4)$ 15-plet mesons $\left(\rho, \rho^{\prime}, a_{1}, b_{1}, h_{1}, \omega, \omega^{\prime}\right)$ are also degenerate. This implies that actually some higher symmetry is observed that includes the $S U(4)$ as a subgroup [12]. It was found that no higher symmetry exists, that would connect local quark bilinears from the 15-plet and singlet of $S U(4)$ within the same irreducible representation [17].

This challenging problem has been solved in ref. [13]. Hadron spectra are extracted from the correlation functions calculated with the gauge-invariant source operators. At each time slice " $t$ " a meson correlator contains minimum the lowest Fock $\bar{q} q$ component with a quark and an antiquark located at different space points $\mathbf{x}$ and y. Both $q$ and $\bar{q}$ interact with the same gauge configuration. Then all arguments of the previous section apply independently for $q$ and $\bar{q}$. Since the $S U\left(2 N_{F}\right)$ invariance is local, we can perform $S U\left(2 N_{F}\right)$ rotations at points $\mathbf{x}$ and $\mathbf{y}$ with different rotation parameters. It is then clear that the meson correlation function with the $\bar{q} q$ valence content has a bilocal $S U\left(2 N_{F}\right) \times S U\left(2 N_{F}\right)$ symmetry. A symmetry of higher Fock components is obviously larger, but the whole correlator has a symmetry of the lowest $\bar{q} q$ component. Obviously, averaging over gauge configurations does not change this symmetry property.

The same argument applies to baryons and in this case we expect a trilocal $S U\left(2 N_{F}\right) \times S U\left(2 N_{F}\right) \times S U\left(2 N_{F}\right)$ symmetry.

One of the irreducible representations of the $S U(4) \times S U(4)$ is 16-dimensional and is a direct sum of the 15-plet and singlet of $S U(4)$. Hence a direct prediction of this bilocal symmetry is a degeneracy of the $S U(4)$ singlet and of the $S U(4)$ 15-plet, in agreement with the lattice observations. This symmetry is bilocal and cannot be represented by the local composite operators which is consistent with conclusions of Ref. [17].

\section{A short summary of our findings.}

Our main findings can be summarized as follows.

1. The classical part of the partition function (the integrand), excluding irrelevant exact zero mode contributions, has $S U(2)_{C S}$ and $S U\left(2 N_{F}\right)$ local symmetries. Since these symmetries are not symmetries of the QCD Lagrangian we refer them as hidden classical symmetries of QCD. There are no respective conserved Noether currents. These symmetries are broken at the quantum level by the axial anomaly and by the quark condensate. The physics of chiral symmetry spontaneous breaking and of anomaly is contained in the near-zero modes of the Dirac operator. Their truncation on the lattice should restore not only the $S U\left(N_{f}\right)_{L} \times S U\left(N_{F}\right)_{R} \times U(1)_{A}$ chiral symmetry but actually higher hidden classical symmetries $S U(2)_{C S}$ and $S U\left(2 N_{F}\right)$.

2. We have shown that elimination of the near-zero modes leads to $S U\left(2 N_{F}\right) \times S U\left(2 N_{F}\right)$ and $S U\left(2 N_{F}\right) \times$ $S U\left(2 N_{F}\right) \times S U\left(2 N_{F}\right)$ symmetries in mesons and baryons.

3. The bilocal $S U(4) \times S U(4)$ symmetry explains a degeneracy of the $S U(4)$ singlet $f_{1}$ correlator with the $S U(4)$ 15-plet $\rho, \rho^{\prime}, \omega, \omega^{\prime}, h_{1}, a_{1}, b_{1}$ correlators. 


\section{Implications}

It is natural to expect many different implications of the hidden classical symmetry. Here we will mention a most dramatic one [18].

At high temperature the quark condensate of the vacuum vanishes. There are lattice indications that above the critical temperature the $U(1)_{A}$ symmetry is restored and a gap opens in the Dirac spectrum $[19,20] .{ }^{3}$ Then it follows that the $S U(2)_{C S}$ and $S U\left(2 N_{F}\right)$ symmetries are manifest in Euclidean correlation functions and observables. Such symmetries cannot be obtained in terms of deconfined quarks and gluons in Minkowski space, where we live. Hence at high temperatures QCD is also in the confining regime and elementary objects are color singlet $S U(4)$ symmetric "hadrons". "Hadrons" with such a symmetry can be directly constructed in Minkowski space [23].

We acknowledge a partial support from the Austrian Science Fund (FWF) through the grant P26627-N27.

\section{References}

[1] K. Fujikawa, Phys. Rev. D 212848 (1980). Erratum: [Phys. Rev. D 221499 (1980)].

[2] T. Banks and A. Casher, Nucl. Phys. B 169103 (1980).

[3] M. Denissenya, L. Y. Glozman and C. B. Lang, Phys. Rev. D 89077502 (2014).

[4] M. Denissenya, L. Y. Glozman and C. B. Lang, Phys. Rev. D 91034505 (2015).

[5] M. Denissenya, L. Y. Glozman and M. Pak, Phys. Rev. D 91114512 (2015).

[6] M. Denissenya, L. Y. Glozman and M. Pak, Phys. Rev. D 92074508 (2015).

[7] G. 't Hooft, Phys. Rept. 142357 (1986).

[8] E. V. Shuryak, Nucl. Phys. B 20393 (1982).

[9] D. Diakonov and V. Y. Petrov, Nucl. Phys. B 272457 (1986).

[10] T. Schäfer and E. V. Shuryak, Rev. Mod. Phys. 70323 (1998).

[11] L. Y. Glozman, Eur. Phys. J. A 5127 (2015).

[12] L. Y. Glozman and M. Pak, Phys. Rev. D 92016001 (2015).

[13] L. Y. Glozman, arXiv:1511.05857 [hep-ph].

[14] H. Leutwyler and A. V. Smilga, Phys. Rev. D 465607 (1992).

[15] R. Brower, S. Chandrasekharan, J. W. Negele and U. J. Wiese, Phys. Lett. B 56064 (2003).

[16] S. Aoki, H. Fukaya and Y. Taniguchi, Phys. Rev. D 86114512 (2012).

[17] T. D. Cohen, Phys. Rev. D 93034508 (2016).

[18] L. Y. Glozman, arXiv:1512.06703 [hep-ph].

[19] G. Cossu, S. Aoki, H. Fukaya, S. Hashimoto, T. Kaneko, H. Matsufuru and J. I. Noaki, Phys. Rev. D 87, no. 11, 114514 (2013) [Phys. Rev. D 88, no. 1, 019901 (2013)].

[20] A. Tomiya, G. Cossu, H. Fukaya, S. Hashimoto and J. Noaki, PoS LATTICE 2014, 211 (2015) [arXiv:1412.7306 [hep-lat]]; G. Cossu et al. [JLQCD Collaboration], arXiv:1511.05691 [hep-lat];

[21] S. Sharma, V. Dick, F. Karsch, E. Laermann and S. Mukherjee, arXiv:1602.02197 [hep-lat].

[22] S. Aoki [JLQCD Collaboration], PoS(CD15) 045 (2015) [arXiv:1603.00997 [hep-lat]].

[23] M. Shifman, Phys. Rev. D 93, no. 7, 074035 (2016).

\footnotetext{
${ }^{3}$ See also the opposite statement [21] and its critique [22].
} 